\section{Stabilizing Linear Model Predictive Control Under Inexact Numerical Optimization}

Matteo Rubagotti, Panagiotis Patrinos, and Alberto Bemporad

\begin{abstract}
This note describes a model predictive control (MPC) formulation for discrete-time linear systems with hard constraints on control and state variables, under the assumption that the solution of the associated quadratic program is neither optimal nor satisfies the inequality constraints. This is common in embedded control applications, for which real-time constraints and limited computing resources dictate restrictions on the possible number of on-line iterations that can be performed within a sampling period. The proposed approach is rather general, in that it does not refer to a particular optimization algorithm, and is based on the definition of an alternative MPC problem that we assume can only be solved within bounded levels of suboptimality, and violation of the inequality constraints. By showing that the inexact solution is a feasible suboptimal one for the original problem, asymptotic or exponential stability is guaranteed for the closed-loop system. Based on the above general results, we focus on a specific dual accelerated gradient-projection method to obtain a stabilizing MPC law that only requires a predetermined maximum number of on-line iterations.
\end{abstract}

Index Terms-Model predictive control, real-time control, embedded control, numerical optimization.

\section{INTRODUCTION}

The use of MPC [2] has recently expanded from traditional applications in the process industry to fields like mechatronics, automotive, and aerospace, thanks to the increasingly available computing power, and to fast optimization algorithms. The most common formulation of an MPC problem is based on a linear model, linear constraints on inputs and states, and quadratic stage and terminal costs. The resulting optimization problem to be solved on line is translated into a quadratic program (QP), for which fast solvers are available, such as active-set methods [3], [4], interior-point methods [5], [6] and semismooth Newton methods [7]. However, one of the main issues in the practical implementation of embedded controllers is the certification of the worst-case execution time. The recent research on real-time MPC aims at designing optimization algorithms that give an acceptable suboptimal solution in an a-priori bounded number of iterations, for which the bounds are much tighter than for the previously-mentioned solvers (see, e.g., [8, Section IA]). To achieve this goal, different variants of fast gradient methods, first proposed by Nesterov [9], [10] have been applied to MPC [8], [11], [12]. In [13] the authors proposed an accelerated dual gradient projection method based on [9], called GPAD (see also [14]-[16]). Although GPAD is a dual method, bounds on the maximum number of iterations required to achieve specified levels of primal suboptimality and constraint violation are provided as complexity certificates. In case a primal method is used (e.g., [8]), the suboptimal solution will not violate the inequality constraints, and closed-loop stability can be proved [17]. On the other hand, dual methods (e.g., [15]) can be successfully applied to more general MPC formulations (with polytopic mixed constraints on states and inputs), but they have the drawback of providing inexact solutions (i.e., the inequality constraints are violated) for the primal problem. Solutions to this drawback (which, however, do not provide a-priori bounds on the maximum number of iterations valid for the entire region of attraction) were recently proposed in

M. Rubagotti is with Nazarbayev University (NU), 53 Kabanbay Batyr Ave, 010000 Astana, Kazakhstan. Email: matteo.rubagotti@nu.edu.kz. P. Patrinos and A. Bemporad are with IMT - Institute for Advanced Studies, Piazza San Ponziano 6, 55100 Lucca, Italy. Email: \{panagiotis.patrinos, alberto.bemporad\} @imtlucca.it.

A preliminary version of this note can be found in [1]. This work was partially supported by NU seed grant "Real-time model predictive control".
[18], [19]. The main contribution of the present work is to prove asymptotic/exponential stability of closed-loop systems in a given domain of attraction in case an optimization solver is used that only gives a solution within prescribed tolerances on suboptimality and constraints violation. These are typically related to the maximum number of iterations that can be executed within a sampling period, such as in the algorithms of [15], [16]. Taking the selected tolerances into account, the original MPC problem is modified so that stability is enforced by design, in spite of the possible low quality of the solution, at the same time guaranteeing bounds on performance loss with respect to the standard MPC formulation (i.e., the problem one would solve exactly).

\section{BASIC NOTATION}

Let $\mathbb{R}_{>0}, \mathbb{R}_{\geq 0}, \mathbb{N}_{>0}$ and $\mathbb{N}_{\geq 0}$ denote the sets of positive reals, non-negative reals, positive integers and non-negative integers, respectively. Given two integers $a \leq b$, let $\mathbb{N}_{[a, b]} \triangleq\{a, a+1, \ldots, b\}$, and $\mathbb{N}_{b} \triangleq\{0,1, \ldots, b\}$. Given $v \in \mathbb{R}^{n}$, let $\|v\|$ denote its Euclidean norm. Given $u, v \in \mathbb{R}^{n}$, the notation $u \leq v$ refers to componentwise inequalities. Given $M \in \mathbb{R}^{n \times n}, M^{\prime}$ is its transpose, $\rho(M)$ its spectral radius. We write $M \succeq 0(M \succ 0)$ if $M$ is symmetric positive semidefinite (positive definite). For $M \in \mathbb{R}^{n \times n}$, we use $\lambda_{\min }(M)$ and $\lambda_{\max }(M)$ to indicate its minimum and maximum eigenvalues, respectively. Also, $\mathbf{1}_{n} \triangleq\left[\begin{array}{lll}1 & \ldots & 1\end{array}\right]^{\prime} \in \mathbb{R}^{n}$. Given a set $\mathcal{X} \subseteq \mathbb{R}^{n}$, its interior is denoted by $\operatorname{int}(\mathcal{X})$. Given $\lambda \in \mathbb{R}_{\geq 0}$, we define $\lambda \mathcal{X} \triangleq\left\{x \in \mathbb{R}^{n}: x=\lambda y, y \in \mathcal{X}\right\}$.

\section{STANDARD MPC FORMULATION}

Consider the discrete-time LTI state-space model

$$
x(t+1)=A x(t)+B u(t)
$$

where $t \in \mathbb{N}_{\geq 0}, x \in \mathbb{R}^{n_{x}}, u \in \mathbb{R}^{n_{u}}$, and the state vector $x$ is assumed to be available for feedback. The state and input values can be represented in a single vector

$$
z(t) \triangleq\left[\begin{array}{l}
x(t) \\
u(t)
\end{array}\right] \in \mathbb{R}^{n_{z}}, n_{z} \triangleq n_{x}+n_{u}
$$

and are required to satisfy the constraint

$$
z(t) \in \mathcal{Z} \triangleq\left\{z \in \mathbb{R}^{n_{z}} \mid F_{z} z \leq \mathbf{1}_{s_{z}}\right\}
$$

with $F_{z}=\left[\begin{array}{ll}F & G\end{array}\right]$, and $F \in \mathbb{R}^{s_{z} \times n_{x}}, G \in \mathbb{R}^{s_{z} \times n_{u}}, s_{z} \in \mathbb{N}_{>0}$. Note that (2) implies that $\mathcal{Z}$ is nonempty, closed, and $0 \in \operatorname{int}(\mathcal{Z})$. We additionally require $\mathcal{Z}$ to be compact. The representation of $\mathcal{Z}$ in (2) is without loss of generality [20], since it can represent any polytope that contains the origin in its interior.

The problem of regulating $x(t)$ to the origin while satisfying (2) point-wise in time can be solved by a standard MPC law for linear systems. In particular, the procedure described in the remainder of this section is a starting point for the subsequent theoretical development. Given two weight matrices $Q=Q^{\prime} \in \mathbb{R}^{n_{x} \times n_{x}}$ and $R=R^{\prime} \in$ $\mathbb{R}^{n_{u} \times n_{u}}$, we define the stage cost $\ell(x, u) \triangleq \frac{1}{2}\left(x^{\prime} Q x+u^{\prime} R u\right)$.

Assumption 1: Matrices $A, B, Q, R$ satisfy one of the following:

(a) The pair $(A, B)$ is stabilizable, $Q \succ 0$, and $R \succ 0$.

(b) The pair $(A, B)$ is stabilizable, $Q \succeq 0$ is such that $C^{\prime} C=Q$, $C \in \mathbb{R}^{n_{y} \times n_{x}}$, the pair $(A, C)$ is detectable, $R \succ 0$.

Clearly, Assumption 1a is more restrictive than Assumption 1b. In both cases, we define the linear auxiliary control law, $\kappa(x) \triangleq K x$, where $K \in \mathbb{R}^{n_{u} \times n_{x}}$ is the gain associated to the infinite-horizon linear quadratic regulator (IH-LQR) defined by matrices $A, B, Q$, and $R$. By applying $u(t)=\kappa(x(t))$, we obtain the closed-loop system

$$
x(t+1)=A_{K} x(t),
$$


where $A_{K} \triangleq A+B K$. By the theory of IH-LQR, Assumption 1 implies $\rho\left(A_{K}\right)<1$. The terminal cost is defined as $V_{f}(x) \triangleq \frac{1}{2} x^{\prime} P x$, where $P=P^{\prime} \succ 0$ is the solution of the algebraic Riccati equation associated with the IH-LQR. Finally, we define the terminal set

$$
\mathcal{X}_{f} \triangleq\left\{x \in \mathbb{R}^{n_{x}} \mid F_{f} x \leq \mathbf{1}_{s_{f}}\right\},
$$

with $F_{f} \in \mathbb{R}^{s_{f} \times n_{x}}, s_{f} \in \mathbb{N}_{>0}$, which is assumed to be a (not necessarily maximal) positively invariant set in

$$
\mathcal{X}_{K} \triangleq\left\{x \in \mathbb{R}^{n_{x}} \mid(F+G K) x \leq \mathbf{1}_{s_{z}}\right\},
$$

for the closed-loop system (3), i.e.,

$$
x \in \mathcal{X}_{f} \Rightarrow\left[\begin{array}{c}
x \\
K x
\end{array}\right] \in \mathcal{Z}, A_{K} x \in \mathcal{X}_{f}
$$

The MPC law is determined by solving the optimization problem

$$
V^{\star}(x)=\min _{\mathbf{z}}\left\{V_{N}(\mathbf{z}) \mid \mathbf{z} \in \mathcal{S}_{N}(x)\right\},
$$

where $\mathbf{z} \triangleq\left[\begin{array}{llll}z_{0}^{\prime} & \cdots & z_{N-1}^{\prime} & x_{N}^{\prime}\end{array}\right]^{\prime}, z_{k} \triangleq\left[\begin{array}{ll}x_{k}^{\prime} & u_{k}^{\prime}\end{array}\right]^{\prime}$. The finitehorizon cost function is

$$
V_{N}(\mathbf{z})=\sum_{k=0}^{N-1} \ell\left(x_{k}, u_{k}\right)+V_{f}\left(x_{N}\right),
$$

the parametric constraint set is given by

$$
\begin{gathered}
\mathcal{S}_{N}(x)=\left\{\mathbf{z} \in \mathcal{A}(x) \mid z_{k} \in \mathcal{Z}, k \in \mathbb{N}_{N-1}, x_{N} \in \mathcal{X}_{f}\right\}, \\
\mathcal{A}(x)=\left\{\mathbf{z} \mid x_{0}=x, x_{k+1}=A x_{k}+B u_{k}, k \in \mathbb{N}_{N-1}\right\},
\end{gathered}
$$

while $N \in \mathbb{N}_{>0}$ is the prediction horizon. The set of states $x$ for which a feasible solution of (7) with prediction horizon $N$ exists is referred to as $\mathcal{D}_{N}$. It is well known that for $x \in \mathcal{D}_{N}$, the (unique) optimal state-input sequence $\mathbf{z}^{\star}(x)$, associated to problem (7), can be obtained by solving a quadratic program (QP). Then, according to the receding horizon principle, only the first control move $u_{0}^{\star}(x)$ is applied to the system at time $t$, while the optimization process with the same prediction horizon $N$ is repeated at time $t+1$. If Assumption 1 holds, and the control law $u(t)=u_{0}^{\star}(x(t))$ associated with the optimal sequence $\mathbf{z}^{\star}(x)$ is applied at each $t \in \mathbb{N}_{\geq 0}$, the set $\mathcal{D}_{N}$ is positively invariant for the resulting closed-loop system, and the origin is an asymptotically stable equilibrium point for the closed-loop system with domain of attraction equal to $\mathcal{D}_{N}$ (also exponentially stable if Assumption 1a holds). This result is an extension of results in [2] to the case of mixed constraints.

\section{MPC FORMULATION FOR INEXACT SOLVERS}

The standard MPC formulation requires finding, at each time instant $t$, the optimal solution of (7). In this section we formulate a modification of problem (7) to determine a stabilizing control law even if the solution is suboptimal and violates the constraints, assuming that bounds on both inaccuracies are given. After fixing the maximum constraint violation $\epsilon \in \mathbb{R}_{\geq 0}$, and the maximum level of suboptimality $\delta \in \mathbb{R}_{\geq 0}$ a priori, a problem with tightened constraints, as described in the following, is obtained. Then, given the QP solver, the needed number of iterations required to solve it within the given bounds is determined: typically, the need for smaller values of these parameters leads to a larger number of numerical iterations. Given the available sampling time, the designer can therefore find the maximum allowed number of iterations, so as to reduce the conservativeness and obtain a solution which is as close as possible to that of (7). In Section VI, we provide a detailed explanation of how these parameters are obtained, based on the specific solver presented in [15].

Assumption 2: The parameter $\in \in \mathbb{R}_{\geq 0}$ is chosen so as to satisfy

$$
\epsilon<\min \left\{\frac{1}{N}, 1-\rho\left(A_{K}\right)\right\} .
$$

Given $\epsilon$ and $k \in \mathbb{N}_{[0, N]}$, we define the tightened sets

$$
\mathcal{Z}_{k}^{\epsilon} \triangleq(1-k \epsilon) \mathcal{Z}=\left\{z \in \mathbb{R}^{n_{z}} \mid F_{z} z \leq(1-k \epsilon) \mathbf{1}_{s_{z}}\right\} \subseteq \mathcal{Z} .
$$

Remark 1: The first term in the min in (11) implies that $0 \in$ $\operatorname{int}\left(\mathcal{Z}_{N}^{\epsilon}\right)$, while the second term implies that $\rho\left((1-\epsilon)^{-1} A_{K}\right)<$ 1 , which by [21, Lemma 3], [22, Theorem 2.1], ensures that the maximal $(1-\epsilon)$-contractive set for (3) contains the origin in its interior (see, e.g., [20] for the formal definition of contractive set). As $\rho\left(A_{K}\right)<1$, it is always possible (in principle) to find an $\epsilon$ small enough to satisfy Assumption 2. However, this requirement influences the settings of the numerical solver (typically, more iterations of the solver are required in order to obtain a smaller $\epsilon$ ).

A different terminal set with respect to $\mathcal{X}_{f}$ is defined as

$$
\mathcal{X}_{f}^{\epsilon} \triangleq\left\{x \in \mathbb{R}^{n_{x}} \mid F_{f}^{\epsilon} x \leq \mathbf{1}_{s_{f}^{\epsilon}}\right\}
$$

with $F_{f}^{\epsilon} \in \mathbb{R}^{s_{f}^{\epsilon} \times n_{x}}$ and $s_{f}^{\epsilon} \in \mathbb{N}_{>0}$, and is a (not necessarily maximal) $(1-\epsilon)$-contractive set in

$$
\mathcal{X}_{K}^{\epsilon} \triangleq\left\{x \in \mathbb{R}^{n_{x}} \mid(F+G K) x \leq(1-N \epsilon) \mathbf{1}_{s_{z}}\right\},
$$

for the closed-loop system (3), i.e.,

$$
x \in \mathcal{X}_{f}^{\epsilon} \Rightarrow\left[\begin{array}{c}
x \\
K x
\end{array}\right] \in \mathcal{Z}_{N}^{\epsilon}, A_{K} x \in(1-\epsilon) \mathcal{X}_{f}^{\epsilon}
$$

Notice that, by Assumption 2, such a set exists non-empty. The set $(1-\epsilon) \mathcal{X}_{f}^{\epsilon}$ can be described as

$$
(1-\epsilon) \mathcal{X}_{f}^{\epsilon}=\left\{x \in \mathbb{R}^{n_{x}} \mid F_{f}^{\epsilon} x \leq(1-\epsilon) \mathbf{1}_{s_{f}^{\epsilon}}\right\}
$$

which is analogous to the definition of the sets $\mathcal{Z}_{k}^{\epsilon}$ in (12). The modified finite-horizon optimal control problem takes the form

$$
V_{\epsilon}^{\star}(x)=\min _{\mathbf{z}}\left\{V_{N}(\mathbf{z}) \mid \mathbf{z} \in \mathcal{S}_{N}^{\epsilon}(x)\right\},
$$

where

$\mathcal{S}_{N}^{\epsilon}(x)=\left\{\mathbf{z} \in \mathcal{A}(x) \mid z_{k} \in \mathcal{Z}_{k+1}^{\epsilon}, k \in \mathbb{N}_{N-1}, x_{N} \in(1-\epsilon) \mathcal{X}_{f}^{\epsilon}\right\}$.

The set $\mathcal{D}_{N}^{\epsilon}$ is defined as the set of states $x \in \mathbb{R}^{n_{x}}$ for which there exists a feasible solution of (17). For every $x \in \mathcal{D}_{N}^{\epsilon}$, the unique optimal solution of (17) is denoted by $\mathbf{z}_{\epsilon}^{\star}(x)$.

For every $x \in \mathcal{D}_{N}^{\epsilon}$, we suppose that a vector $\overline{\mathbf{z}}(x) \in \mathbb{R}^{N n_{z}+n_{x}}$ can be computed, satisfying the following assumption:

Assumption 3: For every $x \in \mathcal{D}_{N}^{\epsilon}$, vector $\overline{\mathbf{z}}(x)=$ $\left[\begin{array}{llll}\bar{z}_{0}^{\prime} & \cdots & \bar{z}_{N-1}^{\prime} & \bar{x}_{N}^{\prime}\end{array}\right]^{\prime}$ is such that

$$
\begin{gathered}
V_{N}(\overline{\mathbf{z}}(x))-V_{\epsilon}^{\star}(x) \leq \delta, \\
\overline{\mathbf{z}}(x) \in \mathcal{A}(x), \\
\bar{z}_{k} \in \mathcal{Z}_{k}^{\epsilon}, \quad k \in \mathbb{N}_{N-1}, \\
\bar{x}_{N} \in \mathcal{X}_{f}^{\epsilon}, \\
\overline{\mathbf{z}}(x)=\mathbf{z}_{\epsilon}^{\star}(x), \quad \text { if } x \in \mathcal{X}_{f}^{\epsilon},
\end{gathered}
$$

where $\bar{z}_{k} \triangleq\left[\begin{array}{ll}\bar{x}_{k}^{\prime} & \bar{u}_{k}^{\prime}\end{array}\right]^{\prime}, k \in \mathbb{N}_{N-1}$.

For each $x \in \mathcal{D}_{N}^{\epsilon}$, let $\mathcal{Z}_{\epsilon, \delta}(x)$ denote the set of all vectors $\overline{\mathbf{z}}(x) \in$ $\mathbb{R}^{N n_{z}+n_{x}}$ satisfying conditions (18), and $\mathcal{U}_{\epsilon, \delta}(x)$ the set of all $\bar{u}_{0}(x)$ corresponding to vectors $\overline{\mathbf{z}}(x)$.

Remark 2: Conditions (18c)-(18d) imply that $\overline{\mathbf{z}}(x)$ leads to a violation of each of the $N s_{z}+s_{f}^{\epsilon}$ linear inequalities, $\bar{z}_{k} \in \mathcal{Z}_{k}^{\epsilon}$, $k \in \mathbb{N}_{N-1}, \bar{x}_{N} \in(1-\epsilon) \mathcal{X}_{f}^{\epsilon}$ which is no larger than $\epsilon$. Also, $\mathcal{Z}_{\epsilon, \delta}(x)$ (and consequently $\mathcal{U}_{\epsilon, \delta}(x)$ ) is nonempty for any $x \in \mathcal{D}_{N}^{\epsilon}$, since it contains $\mathbf{z}_{\epsilon}^{\star}(x)$.

We next state a useful result, which follows mainly from (18e) and from well-known results in MPC, and the proof of which is omitted due to space limitation. 
Lemma 1: Let Assumptions 1-3 hold. Then

$$
V_{N}(\overline{\mathbf{z}})=V_{\epsilon}^{\star}(x)=V^{\star}(x)=V_{f}(x)
$$

for all $x \in \mathcal{X}_{f}^{\epsilon}$, and all $\overline{\mathbf{z}} \in \mathcal{Z}_{\epsilon, \delta}(x)$ with $x \in \mathcal{X}_{f}^{\epsilon}$.

Theorem 2: Let Assumptions 1-3 be satisfied, and consider the closed-loop system

$$
x(t+1)=\varphi(x(t))=A x(t)+B \mu(x(t)),
$$

where $\mu(x(t)) \in \mathcal{U}_{\epsilon, \delta}(x(t))$. Then, the following hold:

(i) recursive feasibility for (17) is ensured, i.e., the set $\mathcal{D}_{N}^{\epsilon}$ is a positively invariant set;

(ii) $(x(t), \mu(x(t))) \in \mathcal{Z}, t \in \mathbb{N}_{\geq 0}$;

(iii) if Assumption 1a is satisfied, $x$ converges exponentially to a closed set $\mathcal{F}, 0 \in \mathcal{F} \subseteq \mathcal{D}_{N}^{\epsilon}$, for all $x(0) \in \mathcal{D}_{N}^{\epsilon}$;

(iv) if Assumption 1a is satisfied, and $\delta$ is small enough so that

$$
\mathcal{B}_{\xi} \triangleq\left\{x \in \mathbb{R}^{n_{x}} \mid\|x\|^{2} \leq \frac{2 \delta}{\lambda_{\min }(Q)-\xi}\right\} \subseteq \mathcal{X}_{f}^{\epsilon}
$$

for some $\xi \in \mathbb{R}_{>0}, \xi<\lambda_{\min }(Q)$ (e.g., $\delta=0$ ), the origin is an exponentially stable equilibrium with domain of attraction $\mathcal{D}_{N}^{\epsilon}$;

(v) if Assumption $1 \mathrm{~b}$ is satisfied, and $\delta=0$, the origin is an asymptotically stable equilibrium with domain of attraction $\mathcal{D}_{N}^{\epsilon}$.

Proof: (i) We first prove positive invariance of $\mathcal{D}_{N}^{\epsilon}$ for (20), i.e., $x \in \mathcal{D}_{N}^{\epsilon} \Rightarrow \varphi(x) \in \mathcal{D}_{N}^{\epsilon}$. This can be stated equivalently as $\mathcal{S}_{N}^{\epsilon}(\varphi(x)) \neq \emptyset, \forall x \in \mathcal{D}_{N}^{\epsilon}$ and $\forall \mu(x) \in \mathcal{U}_{\epsilon, \delta}(x)$. Therefore, it suffices to find a vector $\tilde{\mathbf{z}} \in \mathcal{S}_{N}^{\epsilon}(\varphi(x)), \forall x \in \mathcal{D}_{N}^{\epsilon}, \overline{\mathbf{z}}(x) \in \mathcal{Z}_{\epsilon, \delta}(x)$.

Consider any $x \in \mathcal{D}_{N}^{\epsilon}$, along with any $\overline{\mathbf{z}}(x) \in \mathcal{Z}_{\epsilon, \delta}(x)$, where $\overline{\mathbf{z}}(x)=\left[\begin{array}{llll}\bar{z}_{0}^{\prime} & \cdots & \bar{z}_{N-1}^{\prime} & \bar{x}_{N}^{\prime}\end{array}\right]^{\prime}$, with $\bar{z}_{k}=\left[\begin{array}{cc}\bar{x}_{k}^{\prime} & \bar{u}_{k}^{\prime}\end{array}\right]^{\prime}$, $k \in \mathbb{N}_{[0, N-1]}, \mu(x)=\bar{u}_{0}$ and the "shifted" vector $\tilde{\mathbf{z}}=$ $\left[\begin{array}{llll}\tilde{z}_{0}^{\prime} & \cdots & \tilde{z}_{N-1}^{\prime} & \tilde{x}_{N}^{\prime}\end{array}\right]^{\prime}$, where

$$
\begin{aligned}
\tilde{z}_{k} & =\bar{z}_{k+1}, k \in \mathbb{N}_{N-2}, \\
\tilde{z}_{N-1} & =\left[\begin{array}{ll}
\bar{x}_{N}^{\prime} & \left(K \bar{x}_{N}\right)^{\prime}
\end{array}\right]^{\prime}, \\
\tilde{x}_{N} & =A_{K} \bar{x}_{N} .
\end{aligned}
$$

Then, $\tilde{x}_{0}=\bar{x}_{1}=A x+B \mu(x)=\varphi(x)$, where the first equality follows by (22a), the second by (18b), and the third by (20). By (22a) and (18b), $\tilde{x}_{k+1}=\bar{x}_{k+2}=A \bar{x}_{k+1}+B \bar{u}_{k+1}=A \tilde{x}_{k}+B \tilde{u}_{k}$, $k \in \mathbb{N}_{N-2}$. Also, from (22a) and (18c), $\tilde{z}_{k}=\bar{z}_{k+1} \in \mathcal{Z}_{k}^{\epsilon}, k \in$ $\mathbb{N}_{N-2}$. Furthermore, from (18d), $\bar{x}_{N} \in \mathcal{X}_{f}^{\epsilon}$. Using (15), (22b), (22c) we get $\tilde{z}_{N-1} \in \mathcal{X}_{f}^{\epsilon}$ and $\tilde{x}_{N}=A_{K} \bar{x}_{N} \in(1-\epsilon) \mathcal{X}_{f}^{\epsilon}$. Therefore, $\tilde{\mathbf{z}}(x) \in \mathcal{S}_{N}^{\epsilon}(\varphi(x))$.

(ii) Since $\mathcal{D}_{N}^{\epsilon}$ is invariant, and $\mu(x(t)) \in \mathcal{U}_{\epsilon, \delta}(x(t)) \neq \emptyset$, from (18c) one has $\left(x(t), \mu(x(t)) \in \mathcal{Z}_{1}^{\epsilon} \subseteq \mathcal{Z}, t \in \mathbb{N}_{\geq 0}\right.$.

(iii) To prove exponential convergence to a set including the origin (defined as $\mathcal{F}=\left\{x \in \mathbb{R}^{n}:\|x\| \leq \eta\right\}, \eta \in \mathbb{R}_{\geq 0}$ ), it is necessary to prove that there exist $c_{1} \in \mathbb{R}_{>0}$ and $c_{2} \in \mathbb{R}_{[0,1)}$, s.t.

$$
\|x(t)\| \leq c_{1} c_{2}^{t}\left\|x_{0}\right\|+\eta, \forall x(0) \in \mathcal{D}_{N}^{\epsilon}, \forall t \in \mathbb{N}_{\geq 0} .
$$

This is proved by finding a Lyapunov function $V: \mathcal{X} \rightarrow \mathbb{R}$, s.t.

$$
\begin{aligned}
\alpha_{1}\|x\|^{2} & \leq V(x) \leq \alpha_{2}\|x\|^{2}, \\
V(\varphi(x)) & -V(x) \leq-\alpha_{3}\|x\|^{2}+d,
\end{aligned}
$$

where $\alpha_{i} \in \mathbb{R}_{>0}, i=1,2,3$, and $d \in \mathbb{R}_{\geq 0}, \forall x \in \mathcal{D}_{N}^{\epsilon}$. By slightly modifying the results in [23, Theorem 2.5], it is easy to prove that (24a)-(24b) imply (23), with $c_{1}=\sqrt{2 \alpha_{2} / \alpha_{1}}, c_{2}=\sqrt{1-\alpha_{3} / \alpha_{2}}$, $\eta=\sqrt{2 d \alpha_{2} /\left(\alpha_{1} \alpha_{3}\right)}$ (omitted due to space limitation).

Keeping this in mind, we choose $V_{\epsilon}^{\star}$ as candidate Lyapunov function. One can easily obtain that $V_{\epsilon}^{\star}(x) \geq \frac{1}{2} x^{\prime} Q x \geq$ $\frac{1}{2} \lambda_{\min }(Q)\|x\|^{2}, \forall x \in \mathcal{D}_{N}^{\epsilon}$. On the other hand, from Lemma 1 it follows that $V_{\epsilon}^{\star}(x)=V_{f}(x)=\frac{1}{2} x^{\prime} P x \leq \frac{1}{2} \lambda_{\max }(P)\|x\|^{2}, \forall x \in \mathcal{X}_{f}^{\epsilon}$. Following [2, Proposition 2.18], all needed assumptions are satisfied to state that $\exists c \in \mathbb{R}_{\geq 0}$ s.t. $V_{\epsilon}^{\star}(x) \leq \frac{c}{2} \lambda_{\max }(P)\|x\|^{2}, \forall x \in \mathcal{D}_{N}^{\epsilon}$. Condition (24a) is then satisfied with $\alpha_{1}=\frac{1}{2} \lambda_{\min }(Q)$ and $\alpha_{2}=$ $\frac{c}{2} \lambda_{\max }(P), \forall x \in \mathcal{D}_{N}^{\epsilon}$. To prove that also (24b) holds, we recall that $\tilde{\mathbf{z}} \in \mathcal{S}_{N}^{\epsilon}(\varphi(x))$, and then $V_{N}(\tilde{\mathbf{z}}) \geq V_{\epsilon}^{\star}(\varphi(x))$. Then, recalling (18a), we have, $\forall x \in \mathcal{D}_{N}^{\epsilon}$,

$$
\begin{aligned}
V_{\epsilon}^{\star}(\varphi(x))-V_{\epsilon}^{\star}(x) & \leq V_{N}(\tilde{\mathbf{z}})-V_{N}(\overline{\mathbf{z}})+\delta \\
& =-\ell(x, \mu(x))+\ell\left(\bar{x}_{N}, K \bar{x}_{N}\right)-V_{f}\left(\bar{x}_{N}\right) \\
& +V_{f}\left(A_{K} \bar{x}_{N}\right)+\delta \\
& =-\ell(x, \mu(x))+\delta \\
& \leq-\frac{1}{2} \lambda_{\min }(Q)\|x\|^{2}+\delta
\end{aligned}
$$

where the first equality follows by (22), and the second by the fact that $K$ is the gain given by the IH-LQR, which implies $\ell\left(\bar{x}_{N}, K \bar{x}_{N}\right)-$ $V_{f}\left(\bar{x}_{N}\right)+V_{f}\left(A_{K} \bar{x}_{N}\right)=0$ (see, e.g., [2]). As a consequence, (24b) holds with $\alpha_{3}=\frac{1}{2} \lambda_{\min }(Q)$, and $d=\delta$. Therefore, the value of $\eta$ in (23) is known, and $x$ converges exponentially to the set $\mathcal{F}=$ $\left\{x \in \mathcal{D}_{N}^{\epsilon} \mid\|x\|^{2} \leq 4 \delta c \lambda_{\max }(P) / \lambda_{\min }^{2}(Q)\right\}$.

(iv) We distinguish two cases. If $\delta=0$, (27) becomes $V^{\star}(\varphi(x))-$ $V^{\star}(x) \leq-\frac{1}{2} \lambda_{\min }(Q)\|x\|^{2}$, which holds for all $x \in \mathcal{D}_{N}^{\epsilon}$, and then condition (24b) holds with $\alpha_{3}=\frac{1}{2} \lambda_{\min }(Q)$, and $d=0$.

On the other hand, if $\delta>0$ and (21) holds for some $\xi \in \mathbb{R}_{>0}$ one has that, for $x \in \mathcal{D}_{N}^{\epsilon} \backslash \mathcal{B}_{\xi}$, (27) can be written as $V_{\epsilon}^{\star}(\varphi(x))-$ $V_{\epsilon}^{\star}(x) \leq-\frac{1}{2} \xi\|x\|^{2}-\frac{1}{2}\left(\lambda_{\min }(Q)-\xi\right)\|x\|^{2}+\delta \leq-\frac{1}{2} \xi\|x\|^{2}$. Since $\mathcal{B}_{\xi} \subseteq \mathcal{X}_{f}^{\epsilon}$ (and $x \in \mathcal{B}_{\xi}$ implies $\mu(x)=K x$ by Lemma 1), for $x \in \mathcal{B}_{\xi}$ we have $V_{\epsilon}^{\star}(\bar{\varphi}(x))-V_{\epsilon}^{\star}(x)=V_{\epsilon}^{\star}\left(A_{K} x\right)-V_{\epsilon}^{\star}(x)=V_{f}\left(A_{K} \bar{x}_{N}\right)-$ $V_{f}\left(\bar{x}_{N}\right)=-\ell(x, K x) \leq-\frac{1}{2} \lambda_{\min }(Q)\|x\|^{2}$. Being $\xi<\lambda_{\min }(Q)$, condition (24b) is satisfied $\forall x \in \mathcal{D}_{N}^{\epsilon}$, with $\alpha_{3}=\xi / 2$, and $d=0$. In both cases, the fulfillment of (23) follows with $c_{1}=\sqrt{2 \alpha_{2} / \alpha_{1}}$, $c_{2}=\sqrt{1-\alpha_{3} / \alpha_{2}}$ (being $\alpha_{1}=\frac{1}{2} \lambda_{\min }(Q)$ and $\alpha_{2}=\frac{c}{2} \lambda_{\max }(P)$ ), and $\eta=0$, thus proving exponential stability of the origin with domain of attraction $\mathcal{D}_{N}^{\epsilon}$.

(v) Also in this case, $V_{\epsilon}^{\star}(x)=V_{f}(x)=\frac{1}{2} x^{\prime} P x \leq$ $\frac{1}{2} \lambda_{\max }(P)\|x\|^{2}, \forall x \in \mathcal{X}_{f}^{\epsilon}$. Also, $V_{\epsilon}^{\star}(x) \geq \frac{1}{2} x^{\prime} Q x=$ $\frac{1}{2}\|C x\|^{2}, \forall x \in \mathcal{D}_{N}^{\epsilon}$. Analogously to (25) and recalling that $\delta=0$, we get $V_{\epsilon}^{\star}(\varphi(x))-V_{\epsilon}^{\star}(x) \leq-\ell(x, \mu(x)) \leq-\frac{1}{2}\|C x\|^{2}$. Summing up, $V_{\epsilon}^{\star}(x) \geq \frac{1}{2}\|C x\|^{2}$ and $V_{\epsilon}^{\star}(\varphi(x))-V_{\epsilon}^{\star}(x) \leq-\frac{1}{2}\|C x\|^{2}$ for $x \in \mathcal{D}_{N}^{\epsilon}$, and $V_{\epsilon}^{\star}(x) \leq \frac{1}{2} \lambda_{\max }(P)\|x\|^{2}$ for $x \in \mathcal{X}_{f}^{\epsilon}$. In [2, Sec. 2.6], it is proved that, if these equations hold for a generic nonlinear system, and if the system is input/output-to-state stable [2, Def. 2.40] (which is satisfied in our case, since Assumption $1 \mathrm{~b}$ imposes $(A, C)$ detectable) then the origin is an asymptotically stable equilibrium point with domain of attraction $\mathcal{D}_{N}^{\epsilon}$.

Remark 3: Problem (17) can be seen as a way to obtain a feasible suboptimal solution to problem (7), in the presence of known bounds on suboptimality, $\delta$, and on constraint violation, $\epsilon$. Note that, as $\epsilon$ is increased, the imposed contractions of the predicted evolution lead to a progressive shrinking of $\mathcal{D}_{N}^{\epsilon} \subseteq \mathcal{D}_{N}$, which in turn leads to conservative results. This effect is strongly system-dependent: for instance, the shrinking of $\mathcal{D}_{N}^{\epsilon}$ is more dramatic if the uncontrollable part of matrix $A$ has large eigenvalues. On the other hand, as both $\delta$ and $\epsilon$ tend to zero, the solution of problem (17) tends to the solution of problem (7), and $\mathcal{D}_{N}^{\epsilon}$ tends to $\mathcal{D}_{N}$.

\section{PERformance Bounds}

In this section we will derive suboptimality bounds of the finitehorizon cost $V_{N}$, corresponding to a suboptimal MPC law satisfying Assumption 3, compared to the value function $V^{\star}$ of the original MPC problem (7). From these results, performance bounds based on the closed-loop infinite-horizon cost are provided. To be able to perform such a comparison, we require the following: 
Assumption 4: The terminal set $\mathcal{X}_{f}$ in (7) is the maximal positive invariant set for (3) in $\mathcal{X}_{K}$, cf. (5), while the terminal set $\mathcal{X}_{f}^{\epsilon}$ in (17) is the $(1-\epsilon)$-maximal contractive set for (3) in $\mathcal{X}_{K}^{\epsilon}$, cf. (14).

The proof of the next lemma follows by [22, Theorem 4.1], [21, Lemma 3] and it is omitted due to space limitations.

Lemma 3: If Assumptions 1, 2 and 4 hold, then there exist a finite integer $N^{\star}$ such that

$$
\begin{aligned}
& \mathcal{X}_{f}=\left\{x \in \mathbb{R}^{n_{x}} \mid C_{K} A_{K}^{j} x \leq \mathbf{1}_{s_{z}}, j \in \mathbb{N}_{N^{\star}}\right\}, \\
& \mathcal{X}_{f}^{\epsilon}=\left\{x \in \mathbb{R}^{n_{x}} \mid C_{K} A_{K}^{j} x \leq \kappa_{j} \mathbf{1}_{s_{z}}, j \in \mathbb{N}_{N^{\star}}\right\},
\end{aligned}
$$

where $C_{K} \triangleq F+G K$ and $\kappa_{j} \triangleq(1-\epsilon)^{j}(1-N \epsilon)$, for all $\epsilon$ satisfying (11).

We remark that the $(1-\epsilon)$-maximal contractive set for (3) is the maximal positive invariant set for $x_{k+1}=(1-\epsilon)^{-1} A_{K} x_{k}$ [21, Lemma 3], therefore it can be computed using well-known techniques [22, Algorithm 3.1]. If Assumptions 1, 2 and 4 hold, due to Lemma 3 , problem (7) can be expressed as

$$
V^{\star}(x)=\min _{\mathbf{z} \in \mathcal{A}(x)}\left\{\begin{array}{l|l}
V_{N}(\mathbf{z}) & \begin{array}{l}
g\left(z_{k}\right) \leq 0, \quad k \in \mathbb{N}_{N-1} \\
g_{k}\left(x_{N}\right) \leq 0, k \in \mathbb{N}_{\left[N, N+N^{\star}\right]}
\end{array}
\end{array}\right\},
$$

where

$$
\begin{aligned}
g(z) & =F_{z} z-\mathbf{1}_{s_{z}}, \\
g_{k}(x) & =C_{K} A_{K}^{N-k} x-\mathbf{1}_{s_{z}}, k \in \mathbb{N}_{\left[N, N+N^{\star}\right]} .
\end{aligned}
$$

The Lagrangian associated with (29) is

$$
\mathcal{L}(\mathbf{z}, \mathbf{y})=V(\mathbf{z})+\sum_{k=0}^{N-1} g\left(z_{k}\right)^{\prime} y_{k}+\sum_{k=N}^{N+N^{\star}} g_{k}\left(x_{N}\right)^{\prime} y_{k},
$$

while the set of dual optimal solutions of (29) is

$$
\mathcal{Y}^{\star}(x)=\underset{\mathbf{y} \geq 0}{\arg \max } \min _{\mathbf{z} \in \mathcal{A}(x)} \mathcal{L}(\mathbf{z}, \mathbf{y}) .
$$

Similarly, for $\epsilon$ satisfying (11), problem (17) can be expressed as

$$
V_{\epsilon}^{\star}(x)=\min _{\mathbf{z} \in \mathcal{A}(x)}\left\{\begin{array}{l|l}
V_{N}(\mathbf{z}) & \begin{array}{l}
g_{k}^{\epsilon}\left(z_{k}\right) \leq 0, k \in \mathbb{N}_{N-1} \\
g_{k}^{\epsilon}\left(x_{N}\right) \leq 0, k \in \mathbb{N}_{\left[N, N+N^{\star}\right]}
\end{array}
\end{array}\right\},
$$

where

$$
\begin{aligned}
& g_{k}^{\epsilon}(z)=F_{z} z-(1-(k+1) \epsilon) \mathbf{1}_{s_{z}}, k \in \mathbb{N}_{N-1}, \\
& g_{k}^{\epsilon}(x)=C_{K} A_{K}^{N-k} x-\kappa_{k+1-N} \mathbf{1}_{s_{z}}, k \in \mathbb{N}_{\left[N, N+N^{\star}\right]},
\end{aligned}
$$

and with the Lagrangian of (33) being

$$
\mathcal{L}_{\epsilon}(\mathbf{z}, \mathbf{y})=V(\mathbf{z})+\sum_{k=0}^{N-1} g_{k}^{\epsilon}\left(z_{k}\right)^{\prime} y_{k}+\sum_{k=N}^{N+N^{\star}} g_{k}^{\epsilon}\left(x_{N}\right)^{\prime} y_{k},
$$

while the set of dual optimal solutions of (33) is

$$
\mathcal{Y}_{\epsilon}^{\star}(x)=\underset{\mathbf{y} \geq 0}{\arg \max } \min _{\mathbf{z} \in \mathcal{A}(x)} \mathcal{L}_{\epsilon}(\mathbf{z}, \mathbf{y}) .
$$

It is immediate to see that $0 \leq V_{\epsilon}^{\star}(x)-V^{\star}(x)$ for any $x \in \mathcal{D}_{N}^{\epsilon}$, since the set of feasible solutions of (33) is a subset of that of (29). The next theorem provides refined lower and upper bounds on $V_{\epsilon}^{\star}(x)-V^{\star}(x)$.

Theorem 4: Let Assumptions 1, 2 and 4 hold. Then, for any $x \in$ $\mathcal{D}_{N}^{\epsilon}$, and any $\mathbf{y}_{\epsilon}^{\star}(x) \in \mathcal{Y}_{\epsilon}^{\star}(x), \mathbf{y}^{\star}(x) \in \mathcal{Y}^{\star}(x)$,

$$
\epsilon\left\|\mathbf{y}^{\star}(x)\right\|_{1} \leq V_{\epsilon}^{\star}(x)-V^{\star}(x) \leq N \epsilon\left\|\mathbf{y}_{\epsilon}^{\star}(x)\right\|_{1} .
$$

Proof: Problems (29), (33) are convex quadratic programs, thus strong duality holds for any $x \in \mathcal{D}_{N}, x \in \mathcal{D}_{N}^{\epsilon}$, respectively. Therefore, by the Lagrangian Saddle Point Theorem (see e.g., [24, Prop. 6.2.4]) for any $x \in \mathcal{D}_{N}$, the unique solution of (29), $\mathbf{z}^{\star}(x)$, and any $\mathbf{y}^{\star} \in \mathcal{Y}^{\star}(x)$ satisfy the saddle point inequality

$$
\mathcal{L}\left(\mathbf{z}^{\star}(x), \mathbf{y}\right) \leq V^{\star}(x) \leq \mathcal{L}\left(\mathbf{z}, \mathbf{y}^{\star}(x)\right),
$$

for all $\mathbf{z} \in \mathcal{A}(x), \mathbf{y} \geq 0$, while for any $x \in \mathcal{D}_{N}^{\epsilon}$, the unique solution of (33), $\mathbf{z}_{\epsilon}^{\star}(x)$, and any $\mathbf{y}_{\epsilon}^{\star}(x) \in \mathcal{Y}_{\epsilon}^{\star}(x)$ satisfy

$$
\mathcal{L}_{\epsilon}\left(\mathbf{z}_{\epsilon}^{\star}(x), \mathbf{y}\right) \leq V_{\epsilon}^{\star}(x) \leq \mathcal{L}_{\epsilon}\left(\mathbf{z}, \mathbf{y}_{\epsilon}^{\star}(x)\right),
$$

for all $\mathbf{z} \in \mathcal{A}(x), \mathbf{y} \geq 0$. Choose any $x \in \mathcal{D}_{N}^{\epsilon} \subseteq$ $\mathcal{D}_{N}$ and set $\mathbf{y}=\mathbf{y}_{\epsilon}^{\star}(x)=\left[\begin{array}{llll}\left(y_{\epsilon, 0}^{\star}\right)^{\prime} & \cdots & \left(y_{\epsilon, N^{\star}}^{\star}\right)^{\prime}\end{array}\right]^{\prime} \bar{\epsilon}$ $\mathcal{Y}_{\epsilon}^{\star}(x)$ in the left part of (38a), and $\mathbf{z}=\mathbf{z}^{\star}(x)=$ $\left[\begin{array}{llll}\left(z_{0}^{\star}\right)^{\prime} & \cdots & \left(z_{N-1}^{\star}\right)^{\prime} & \left(x_{N}^{\star}\right)^{\prime}\end{array}\right]^{\prime} \in \mathcal{A}(x)$ in the right part of (38b). Then $V_{\epsilon}^{\star}(x)-V^{\star}(x) \leq \mathcal{L}_{\epsilon}\left(\mathbf{z}^{\star}(x), \mathbf{y}_{\epsilon}^{\star}(x)\right)-\mathcal{L}\left(\mathbf{z}^{\star}(x), \mathbf{y}_{\epsilon}^{\star}(x)\right)=$ $\sum_{k=0}^{N-1}\left(g_{k}^{\epsilon}\left(z_{k}^{\star}\right)-g_{k}\left(z_{k}^{\star}\right)\right)^{\prime} y_{\epsilon, k}^{\star}+\sum_{k=N}^{N+N^{\star}}\left(g_{k}^{\epsilon}\left(x_{N}^{\star}\right)-g_{k}\left(x_{N}^{\star}\right)\right)^{\prime} y_{\epsilon, k}^{\star}=$ $\sum_{k=0}^{N-1}(k+1) \epsilon \mathbf{1}_{s_{z}}^{\prime} y_{\epsilon, k}^{\star}+\sum_{k=N}^{N+N^{\star}}\left(1-\kappa_{k+1-N}\right) \mathbf{1}_{s_{z}}^{\prime} y_{\epsilon, k}^{\star} \leq$ $N \epsilon \sum_{k=0}^{N+N^{\star}} \mathbf{1}_{s_{z}}^{\prime} y_{\epsilon, k}^{\star}=N \epsilon\left\|\mathbf{y}_{\epsilon}^{\star}(x)\right\|_{1}$, where the first inequality follows by (38), the first equality by (31), (35), the second equality by (30), (34), the last inequality by $\max _{k \in \mathbb{N}_{N-1}}(k+1) \epsilon=N \epsilon$ and

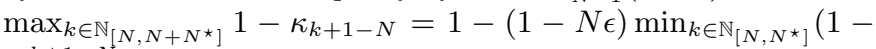
$\epsilon)^{k+1-N} \leq N \epsilon$, and the last equality by the nonnegativity of $\mathbf{y}_{\epsilon}^{\star}(x)$. Hence, the right part of inequality (37) is proved.

To prove the left part, set $\mathbf{z}=\mathbf{z}_{\epsilon}^{\star}(x)=$ $\left[\begin{array}{llll}\left(z_{\epsilon, 0}^{\star}\right)^{\prime} & \cdots & \left(z_{\epsilon, N-1}^{\star}\right)^{\prime} & \left(x_{\epsilon, N}^{\star}\right)^{\prime}\end{array}\right]^{\prime} \in \mathcal{A}(x)$ in the right part of (38a) and $\mathbf{y}=\mathbf{y}_{\epsilon}^{\star}(x)=\left[\begin{array}{lll}\left(y_{0}^{\star}\right)^{\prime} & \cdots & \left(y_{N^{\star}}^{\star}\right)^{\prime}\end{array}\right]^{\prime} \in \mathcal{Y}_{\epsilon}^{\star}(x)$ in the left part of (38b), and follow exactly the same steps to arrive at $V_{\epsilon}^{\star}(x)-V^{\star}(x) \geq \mathcal{L}_{\epsilon}\left(\mathbf{z}_{\epsilon}^{\star}(x), \mathbf{y}^{\star}(x)\right)-\mathcal{L}\left(\mathbf{z}_{\epsilon}^{\star}(x), \mathbf{y}^{\star}(x)\right)=$ $\sum_{k=0}^{N-1}(k+1) \epsilon \mathbf{1}_{s_{z}}^{\prime} y_{k}^{\star}+\sum_{k=N}^{N+N^{\star}}\left(1-\kappa_{k+1-N}\right) \mathbf{1}_{s_{z}}^{\prime} y_{k}^{\star} \geq$ $N \epsilon \sum_{k=0}^{N+N^{\star}} \mathbf{1}_{s_{z}}^{\prime} y_{k}^{\star}=\epsilon\left\|\mathbf{y}^{\star}(x)\right\|_{1}$, where the last inequality follows

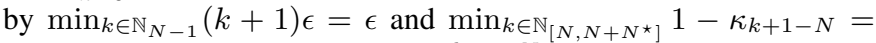
$1-(1-N \epsilon) \max _{k \in \mathbb{N}_{\left[N, N^{\star}\right]}}(1-\epsilon)^{k+1-N}=1-(1-N \epsilon)(1-\epsilon) \geq$ $1-(1-\epsilon)=\epsilon$.

Now we are ready to provide suboptimality estimates for a $\overline{\mathbf{z}}(x) \in$ $\mathcal{Z}_{\epsilon, \delta}(x)$.

Theorem 5: Let Assumptions 1-4 hold. Then, for any $\overline{\mathbf{z}}(x) \in$ $\mathcal{Z}_{\epsilon, \delta}(x)$

$$
V_{N}(\overline{\mathbf{z}}(x))-V^{\star}(x) \leq N \epsilon\left\|y_{\epsilon}^{\star}(x)\right\|_{1}+\delta, x \in \mathcal{D}_{N}^{\epsilon},
$$

for all $\delta$ satisfying (21).

Proof: One has $V_{N}(\overline{\mathbf{z}}(x))-V^{\star}(x)=\left(V_{N}(\overline{\mathbf{z}}(x))-V_{\epsilon}^{\star}(x)\right)+$ $\left(V_{\epsilon}^{\star}(x)-V^{\star}(x)\right) \leq \delta+N \epsilon\left\|y_{\epsilon}^{\star}(x)\right\|_{1}$, where the last equality follows from (18a), (37).

For a feedback law $\mu: \mathbb{R}^{n_{x}} \rightarrow \mathbb{R}^{n_{u}}$, we introduce the infinitehorizon cost corresponding to $\mu$ :

$$
V_{\infty}^{\mu}(x) \triangleq \sum_{t=0}^{\infty} \ell\left(x_{\mu}(t), \mu\left(x_{\mu}(t)\right)\right),
$$

where $x_{\mu}(0)=x, x_{\mu}(t+1)=A x_{\mu}(t)+B \mu\left(x_{\mu}(t)\right)$. The following theorem gives performance bounds for the infinite horizon cost of the system in closed-loop with any $\mu(x) \in \mathcal{U}_{\epsilon, 0}(x)$ in terms of the value function of the original problem.

Theorem 6: Let Assumptions 1-4 hold and $\mu(x) \in \mathcal{U}_{\epsilon, 0}(x)$. Then

$$
\begin{aligned}
& V_{\infty}^{\mu}(x) \leq V^{\star}(x)+N \epsilon\left\|\mathbf{y}_{\epsilon}^{\star}(x)\right\|_{1}, x \in \mathcal{D}_{N}^{\epsilon} \backslash \mathcal{X}_{f}^{\epsilon}, \\
& V_{\infty}^{\mu}(x)=V^{\star}(x), x \in \mathcal{X}_{f}^{\epsilon} .
\end{aligned}
$$

Proof: Since (27) is satisfied with $\delta=0$, by [25, Proposition 2.2], we have $V_{\infty}^{\mu}(x) \leq V_{\epsilon}^{\star}(x)$. Invoking Theorem 4 proves (40a). Equation (40b) follows from Lemma 1 .

\section{DESCRIPTION OF THE OPTIMIZATION ALGORITHM}

In this section we consider and briefly summarize the Dual Accelerated Gradient Projection (GPAD) algorithm proposed in [13] (see also [14], [15]). This algorithm belongs to the category covered by the theoretical results of this note. In particular, it guarantees a bounded $\epsilon$ and $\delta=0$ in a finite number of iterations, and ensures 


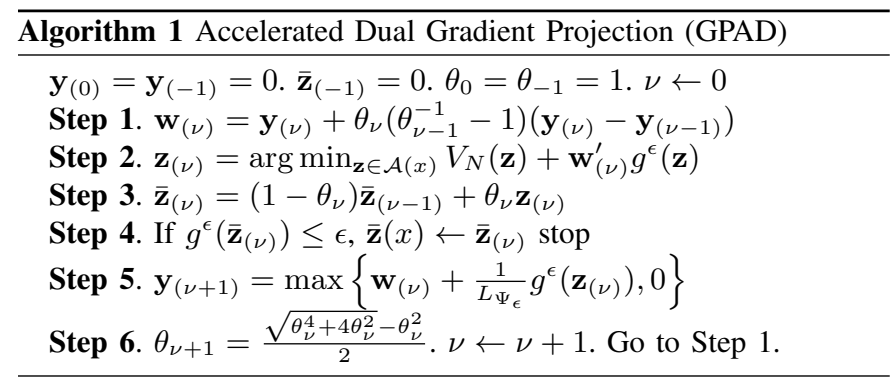

by design that $\mu(x)=K x$ for all $x \in \mathcal{X}_{f}^{\epsilon}$, without the need of employing a dual-mode strategy.

Problem (17) can be expressed as

$$
V_{\epsilon}^{\star}(x)=\min _{\mathbf{z} \in \mathcal{A}(x)}\left\{V_{N}(\mathbf{z}) \mid g^{\epsilon}(\mathbf{z}) \leq 0\right\},
$$

where $g^{\epsilon}(\mathbf{z})=\left(g_{1}^{\epsilon}\left(z_{1}\right), \cdots, g_{N}^{\epsilon}\left(x_{N}\right)\right)$ with $g_{k}^{\epsilon}: \mathbb{R}^{n_{x}+n u} \rightarrow \mathbb{R}^{s_{z}}$, $k \in \mathbb{N}_{N-1}$, defined as $g_{k}^{\epsilon}(z)=F_{z} z-(1-(k+1) \epsilon) \mathbf{1}_{s_{z}}$, while $g_{N}^{\epsilon}(x)=F_{f}^{\epsilon} x-(1-\epsilon) \mathbf{1}_{s_{f}^{\epsilon}}$. The dual function

$$
\Psi_{\epsilon}(\mathbf{y}, x) \triangleq \min _{\mathbf{z} \in \mathcal{A}(x)} V_{N}(\mathbf{z})+g^{\epsilon}(\mathbf{z})^{\prime} y
$$

is Lipschitz-continuous, with Lipschitz constant equal to $L_{\Psi_{\epsilon}}$. The tightest Lipschitz constant for $\nabla \Psi_{\epsilon}$ can be computed by forming explicitly the Hessian of quadratic dual cost $\Psi_{\epsilon}$ as in [14] and calculating its spectral norm. Alternatively, one can either compute the Frobenius or 1-norm of the Hessian of $\Psi_{\epsilon}$ or use the results in [12]. Algorithm 1 is based on the accelerated gradient method of [9] applied to the dual problem $\max _{\mathbf{y} \geq 0} \Psi_{\epsilon}(\mathbf{y}, x)$.

The only complicated part of Algorithm 1 is Step 2. If Problem (41) is posed in condensed form, i.e., the equality constraints corresponding to the state equations have been eliminated (off-line), then Step 2 consists of a matrix-vector product which requires $O\left(N^{2}\right)$ operations. One can do even better, by viewing Step 2 as an unconstrained linear-quadratic optimal control problem, and applying the modified Riccati approach proposed in [13], which requires only $O(N)$ flops to compute $\mathbf{z}_{(\nu)}$.

Note that this is a simplified version of the algorithm proposed in [13]. The following theorem provides an upper bound on the maximum number of iterations to compute a solution satisfying Assumption 3, with $\delta=0$. Since the initial dual iterate is equal to the zero vector, GPAD is doing always better than optimal, so that one has to care only about $\epsilon$-feasibility, and this is the only termination criterion employed at Step 4. The following theorem gives complexity, stability, and performance guarantees for Algorithm 1.

Theorem 7: Let Assumptions 1, 3 and 4 hold. For any $x \in \mathcal{D}_{N}^{\epsilon}$, and $\epsilon$ satisfying (11), Algorithm 1 will terminate after at most

$$
\nu_{\epsilon}^{\star}=\left\lceil\left(\frac{8 L_{\Psi_{\epsilon}} \Delta_{y}^{\epsilon}}{\epsilon}\right)^{\frac{1}{2}}\right\rceil-2
$$

iterations, with $\overline{\mathbf{z}}(x) \in \mathcal{Z}_{\epsilon, 0}(x)$, where

$$
\Delta_{y}^{\epsilon} \triangleq \max _{x \in \mathcal{D}_{N}^{\epsilon}} \min _{\mathbf{y}_{\epsilon}^{\star}(x) \in \mathcal{Y}_{\epsilon}^{\star}(x)}\left\|\mathbf{y}_{\epsilon}^{\star}(x)\right\|_{1}
$$

The corresponding MPC law $\mu(x)=\bar{u}_{0}(x)$ produced by Algorithm 1 renders the origin asymptotically stable (if Assumption 1a holds) or exponentially stable (if Assumption 1b holds) for the closed-loop system (20) with region of attraction $\mathcal{D}_{N}^{\epsilon}$, while the closed-loop infinite-horizon performance satisfies

$$
\begin{aligned}
& V_{\infty}^{\mu}(x) \leq V^{\star}(x)+N \Delta_{y}^{\epsilon} \epsilon, x \in \mathcal{D}_{N}^{\epsilon} \backslash \mathcal{X}_{f}^{\epsilon}, \\
& V_{\infty}^{\mu}(x)=V^{\star}(x), x \in \mathcal{X}_{f}^{\epsilon} .
\end{aligned}
$$

Proof: By Step 2 of Algorithm 1, and linearity of the equality constraints describing $\mathcal{A}(x)$, one has that $\overline{\mathbf{z}}_{(\nu)} \in \mathcal{A}(x)$ for all $\nu \in \mathbb{N}_{\geq 0}$, i.e., requirement (18b) is always fulfilled. In [15, Cor. 6] it is shown that $V_{N}\left(\overline{\mathbf{z}}_{(\nu)}\right) \leq V_{\epsilon}^{\star}(x)$ for all $\nu \in \mathbb{N}_{\geq 0}$, therefore requirement (18a) is fulfilled with $\delta=0$, for every $\nu$. Furthermore, that (18e) is satisfied, comes from the fact that, since $\mathbf{y}_{(0)}=\mathbf{y}_{(-1)}=0$, one has $\mathbf{w}_{(0)}=0$ as well in Step 1. Therefore, $\overline{\mathbf{z}}_{(0)}=\mathbf{z}_{(0)}$ is equal to the solution of the unconstrained problem, which is optimal for (17) if $x \in \mathcal{X}_{f}^{\epsilon}$, due to Lemma 1. In that case, Algorithm 1 terminates at Step 4, for $\nu=0$, since $\overline{\mathbf{z}}_{(0)}$ is feasible for (17). That the algorithm needs no more than $\nu^{\star}$ iterations to achieve $\epsilon$-infeasibility, and thus satisfies requirements $(18 \mathrm{c}),(18 \mathrm{~d})$ is shown in [15, Th. 5, Cor. 7], proving that $\overline{\mathbf{z}}(x) \in \mathcal{Z}_{\epsilon, 0}(x)$. Now, the rest of the statements follow immediately by Theorems 2 and 6 .

Bounds on dual optimal solutions such as the one of (43) are called Uniform Dual Bounds (UDBs) in [13], [15].

Remark 4: According to Theorem 7, GPAD reaches $\epsilon$-feasibility in $O\left(\sqrt{\frac{L_{\Psi_{\epsilon}} \Delta_{y}^{\epsilon}}{\epsilon}}\right)$ iterations, while the complexity estimate to achieve the same level of suboptimality for the dual cost, which is the standard result found in the literature (see, e.g., [9]) is of order $O\left(\sqrt{\frac{L_{\Psi_{\epsilon}}}{\epsilon}} \Delta_{y}^{\epsilon}\right)$, which may be much larger.

Remark 5: The bound on dual optimal solutions, $\Delta_{y}^{\epsilon}$, must be valid on the entire $\mathcal{D}_{N}^{\epsilon}$, in order to be able to guarantee stability and invariance of $\mathcal{D}_{N}^{\epsilon}$ for the closed-loop system. In [15, Sec. VI.B], it is shown that a tight upper bound to $\Delta_{y}^{\epsilon}$ can be computed by solving a Linear Program with Linear Complementarity Constraints (LPLCC) for which specialized efficient algorithms exist for its solution. However, the time needed to solve the resulting LPLCC may grow rapidly with the dimension of the problem. Notice that the techniques proposed in [12], [13], lead to bounds which are valid only on a subset interior of $\mathcal{D}_{N}^{\epsilon}$, since they are based on Slater's condition, and thus cannot be used to derive an iteration bound on the entire $\mathcal{D}_{N}^{\epsilon}$.

\section{Simulation EXAMPLES}

\section{A. Masses Example}

The setup for this example is similar to [6]. It consists of a sequence of $M$ objects of equal mass $m$ serially connected to each other, by spring-dampers of equal spring constant $k$ and damping coefficient $c$, and to walls on either side. Between two consecutive masses there is an actuator exerting tensions. The discrete-time LTI model is obtained after discretization with sampling period of $0.5 \mathrm{~s}$, and it consists of $n_{x}=2 M$ states and $n_{u}=M-1$ inputs. Each state of the system is constrained to lie in $[-4,4]$, while each input in $[-1,1]$. The weight matrices are $Q=I_{n_{x}}, R=I_{n_{u}}$ and the length of the horizon is $N=15$. Table I gives bounds on the maximum number of iterations on $\mathcal{D}_{N}^{\epsilon}$, according to Theorem 7, for number of masses $M=\{2,3\}$ and accuracy $\epsilon \in\left\{10^{-3}, 5 \times 10^{-3}, 10^{-2}\right\}$. For each value of $\epsilon, \nu_{\epsilon}^{\star}$ is the theoretical bound given by Eq. (42), with a tight upper bound on $\Delta_{y}^{\epsilon}$ (cf. (43)) computed by solving the LPLCC described in [15, Sec. VI.B], while $\hat{\nu}_{\epsilon}^{\star}$ is the maximum number of iterations encountered by simulating the closed-loop system from 500 random initial states belonging to $\mathcal{D}_{N}^{\epsilon}$. One can observe that the theoretical bound for the specific example is quite tight. Furthermore in $I$, the theoretical bound for the infinite horizon performance of the closed-loop system, given by Theorem 6, is compared against the practical one, which is the one obtained by simulating the system in closed-loop with Algorithm 1. Specifically, the theoretical relative performance bound $\Delta V_{\epsilon} \triangleq \max _{i \in \mathbb{N}_{[1,100]}}\left\{\frac{N\left\|y\left(x_{0}^{i}\right)\right\|_{1} \epsilon}{V^{\star}\left(x_{0}^{i}\right)}\right\}$ is compared against the measured relative bound given by 
TABLE I: Complexity certification analysis $\left(\nu^{\star}=\right.$ theoretical iteration bound, $\hat{\nu}^{\star}=$ measured iteration bound) and performance bounds for the masses example

\begin{tabular}{|c|cc|cc|cc|}
\hline$M$ & \multicolumn{7}{|c|}{$\epsilon$} \\
\hline & \multicolumn{2}{|c|}{$10^{-3}$} & \multicolumn{2}{|c|}{$5 \times 10^{-3}$} & \multicolumn{2}{c|}{$10^{-2}$} \\
\hline & $\nu_{\epsilon}^{\star}$ & $\hat{\nu}_{\epsilon}^{\star}$ & $\nu_{\epsilon}^{\star}$ & $\hat{\nu}_{\epsilon}^{\star}$ & $\nu_{\epsilon}^{\star}$ & $\hat{\nu}_{\epsilon}^{\star}$ \\
\hline 2 & 1229 & 1025 & 551 & 458 & 389 & 323 \\
3 & 2979 & 2266 & 1332 & 800 & 949 & 584 \\
\hline & $\Delta V_{\epsilon} \%$ & $\Delta \hat{V}_{\epsilon} \%$ & $\Delta V_{\epsilon} \%$ & $\Delta \hat{V}_{\epsilon} \%$ & $\Delta V_{\epsilon} \%$ & $\Delta \hat{V}_{\epsilon} \%$ \\
\hline 2 & 2 & 0.1 & 7.1 & 0.5 & 6.8 & 0.7 \\
3 & 4.8 & 0.3 & 7.7 & 0.5 & 13.6 & 1 \\
\hline
\end{tabular}

TABLE II: Complexity certification analysis $\left(\nu^{\star}=\right.$ theoretical iteration bound, $\hat{\nu}^{\star}=$ measured iteration bound)

\begin{tabular}{|c|ccc|ccc|}
\hline$\epsilon$ & \multicolumn{3}{|c|}{$\nu_{\epsilon}^{\star}$} & \multicolumn{3}{c|}{$\hat{\nu}_{\epsilon}^{\star}$} \\
\hline & \multicolumn{3}{|c|}{ quantile } & \multicolumn{3}{c|}{ quantile } \\
& $25 \%$ & $50 \%$ & $75 \%$ & $25 \%$ & $50 \%$ & $75 \%$ \\
\hline $10^{-3}$ & 1416 & 4154 & 9390 & 804 & 1832 & 3367 \\
\hline $5 \times 10^{-3}$ & 622 & 1853 & 4190 & 352 & 664 & 1149 \\
\hline $10^{-2}$ & 430 & 1307 & 2959 & 236 & 399 & 544 \\
\hline $5 \times 10^{-2}$ & 183 & 613 & 1357 & 75 & 102 & 117 \\
\hline
\end{tabular}

$\Delta \hat{V}_{\epsilon} \triangleq \max _{i \in \mathbb{N}_{[1,100]}}\left\{\frac{V_{\infty}^{\mu}\left(x_{0}^{i}\right)-V^{\star}\left(x_{0}^{i}\right)}{V^{\star}\left(x_{0}^{i}\right)}\right\}$. Another significant conclusion that can be drawn from Table I is that the iteration bounds $\nu_{\epsilon}^{\star}$, $\hat{\nu}_{\epsilon}^{\star}$ decrease as $\epsilon$ increases. For embedded applications, this means that according to hardware specifications and sampling time, one can select the appropriate value of $\epsilon$ that will guarantee stability and invariance of the corresponding closed-loop system. However, the price to pay is a smaller $\mathcal{D}_{N}^{\epsilon}$, and performance degradation, which however appears to be insignificant according to Table I.

\section{B. Random Systems}

The next example presents results for 50 random systems, with $n_{x}=2, n_{u}=1, Q=I_{n_{x}}, R=I_{n_{u}}, N=5$, input constraints $|u| \leq 1$, and mixed constraints $\|y\|_{\infty} \leq 1$, being $y=C x+D u \in \mathbb{R}^{2}$. Table II gives results on the maximum number of iterations on $\mathcal{D}_{N}^{\epsilon}$, according to Theorem 7 , for accuracy $\epsilon \in\left\{10^{-3}, 5 \times 10^{-3}, 10^{-2}, 5 \times\right.$ $\left.10^{-2}\right\}$. As before, for each value of $\epsilon, \nu_{\epsilon}^{\star}$ is the theoretical bound and $\hat{\nu}_{\epsilon}^{\star}$ is the maximum number of iterations encountered by simulating the closed-loop system from 500 random initial states belonging to $\mathcal{D}_{N}^{\epsilon}$. The corresponding quartiles of $\nu_{\epsilon}^{\star}, \hat{\nu}_{\epsilon}^{\star}$ are presented in Table II. Two main observations are in order. The number of iterations decreases as $\epsilon$ decreases, as in the previous example, while the theoretical bound becomes less tight as $\epsilon$ decreases.

Additional simulation examples can be found in [1].

\section{CONCLUSIONS}

This note has proposed an MPC formulation for constrained linear systems with guaranteed stability despite the use of inexact QP solvers. Given the optimal control problem, the QP solver is applied to a modified problem with tightened constraints, so as to obtain a suboptimal solution of the original problem that enjoys guarantees of recursive feasibility and asymptotic (or exponential) stability in a finite and a-priori determined number of iterations. Performance bounds are obtained to analyze the level of suboptimality of the computed solution with respect to the optimal solution of the original problem. Simulation examples show the potential of the proposed approach under hard real-time constraints when a simple dual gradient projection method (GPAD) is used to solve QPs.

\section{REFERENCES}

[1] M. Rubagotti, P. Patrinos, and A. Bemporad, "Stabilizing embedded MPC with computational complexity guarantees," in Europ. Contr. Conf., Zurich, Switzerland, Jul. 2013, pp. 3065-3070.

[2] J. B. Rawlings and D. Q. Mayne, Model Predictive Control: Theory and Design. Nob Hill Publishing, 2009.

[3] R. Bartlett and L. Biegler, "QPSchur: A dual, active-set, schurcomplement method for large-scale and structured convex quadratic programming," Optim. Eng., vol. 7, no. 1, pp. 5-32, 2006.

[4] H. J. Ferreau, H. G. Bock, and M. Diehl, "An online active set strategy to overcome the limitations of explicit MPC," Int. J. Rob. Nonlin. Contr. vol. 18, no. 8, pp. 816-830, 2008.

[5] Y. Wang and S. Boyd, "Fast model predictive control using online optimization," IEEE T. Contr. Sys. Tech., vol. 18, no. 2, pp. 267-278, 2010.

[6] A. Domahidi, A. U. Zgraggen, M. N. Zeilinger, M. Morari, and C. N. Jones, "Efficient interior point methods for multistage problems arising in receding horizon control," in Conf. Dec. Contr., Maui, HI, Dec. 2012, pp. 668-674.

[7] P. Patrinos, P. Sopasakis, and H. Sarimveis, "A global piecewise smooth Newton method for fast large-scale model predictive control," Automatica, vol. 47, no. 9, pp. 2016-2022, 2011.

[8] S. Richter, C. N. Jones, and M. Morari, "Computational complexity certification for real-time MPC with input constraints based on the fast gradient method," IEEE T. Autom. Contr., vol. 57, no. 6, pp. 1391-1403, 2012.

[9] Y. Nesterov, "A method of solving a convex programming problem with convergence rate $O\left(1 / k^{2}\right)$," in Soviet Mathematics Doklady, vol. 27, no. 2, 1983, pp. 372-376.

[10] - Introductory lectures on convex optimization: A basic course. Kluwer Academic Publishers, 2004.

[11] I. Necoara and J. Suykens, "Application of a smoothing technique to decomposition in convex optimization," IEEE T. Autom. Contr., vol. 53 , no. 11 , pp. 2674-2679, 2008.

[12] S. Richter, M. Morari, and C. N. Jones, "Towards computational complexity certification for constrained MPC based on Lagrange relaxation and the fast gradient method," in Conf. Dec. Contr., Orlando, FL, Dec. 2011, pp. 5223-5229.

[13] P. Patrinos and A. Bemporad, "An accelerated dual gradient-projection algorithm for linear model predictive control," in Conf. Dec. Contr. Maui, HI, Dec. 2012, pp. 662-667.

[14] A. Bemporad and P. Patrinos, "Simple and certifiable quadratic programming algorithms for embedded linear model predictive control," in Conf. Nonlin. MPC, Noordwijkerhout, Netherlands, Aug. 2012, pp. 14-20.

[15] P. Patrinos and A. Bemporad, "An accelerated dual gradient-projection algorithm for embedded linear model predictive control," IEEE T. Autom. Contr., 2013, in press.

[16] P. Patrinos, A. Guiggiani, and A. Bemporad, "Fixed-point dual gradient projection for embedded model predictive control," in Europ. Contr. Conf., Zurich, Switzerland, Jul. 2013, pp. 3602-3607.

[17] L. K. McGovern and E. Feron, "Closed-loop stability of systems driven by real-time, dynamic optimization algorithms," in Conf. Dec. Contr. Phoenix, AZ, Dec. 1999, pp. 3690-3696.

[18] M. D. Doan, T. Keviczky, and B. De Schutter, "A distributed optimization-based approach for hierarchical MPC of large-scale systems with coupled dynamics and constraint," in Conf. Dec. Contr., Orlando, FL, Dec. 2011, pp. 5236-5241.

[19] I. Necoara and V. Nedelcu, "Rate analysis of inexact dual first order methods: application to distributed MPC for network systems," arXiv:1302.3129v1, 2013.

[20] F. Blanchini, "Set invariance in control," Automatica, vol. 35, no. 11, pp. 1747-1767, 1999.

[21] A. Bemporad, A. Oliveri, T. Poggi, and M. Storace, "Ultra-fast stabilizing model predictive control via canonical piecewise affine approximations," IEEE T. Autom. Contr., vol. 56, no. 12, pp. 2883-2897, 2011.

[22] E. G. Gilbert and K. T. Tan, "Linear systems with state and control constraints: The theory and application of maximal output admissible sets," IEEE T. Autom. Contr., vol. 36, no. 9, pp. 1008-1020, 1991.

[23] M. Lazar, D. Munoz de la Pena, W. P. M. H. Heemels, and T. Alamo, "On input-to-state stability of min-max nonlinear model predictive control," Sys. Contr. Lett., vol. 57, no. 1, pp. 39-48, 2008.

[24] D. Bertsekas, A. Nedic, and A. Ozdaglar, Convex analysis and optimization. Athena Scientific, 2003.

[25] L. Grune and A. Rantzer, "On the infinite horizon performance of receding horizon controllers," IEEE T. Autom. Contr., vol. 53, no. 9, pp. 2100-2111, 2008. 